\title{
Condensation on intraocular lenses during vitrectomy: effect of perfluorocarbon liquids
}

Yann J. Dacquay ${ }^{1}$, Joseph R. Lee ${ }^{2}$, Andrea Govetto ${ }^{1}$, Matthias Elgeti ${ }^{3}$, Wayne L Hubbell $^{3}$, Pirouz Kavehpour ${ }^{2}$, Jean-Pierre Hubschman ${ }^{1}$

${ }^{1}$ Retina Division, Stein Eye Institute, David Geffen School of Medicine, University of California, Los Angeles, Los Angeles, CA, USA; ${ }^{2}$ UCLA Engineering, Mechanical and Aerospace Engineering, University of California, Los Angeles, Los Angeles, CA, USA; ${ }^{3}$ Department for Chemistry and Biochemistry, Stein Eye Institute, David Geffen School of Medicine, University of California, Los Angeles, Los Angeles, CA, USA

\section{Abstract}

Purpose: To evaluate whether perfluorocarbon (PFO) liquids can exacerbate condensation on intraocular lenses (IOL).

Methods: Two separate experiments were carried out. In the first experiment, a two-segment chamber was constructed out of glass and acrylic panels to serve as an in-vitro eye model. The chamber was placed on a non-activated cooling plate with two IOLs of the same material, one in each segment. $2.0 \mathrm{ml}$ of PFO and/or water for the control were placed in the respective receptacle. The cooling plate was then activated to the desired temperatures. Condensation on the lenses was visually assessed via high-definition ultra-zoom camera by trained observers for three temperatures and three IOL materials.

In the second experiment, Fourier transform infrared (FTIR) spectroscopy was employed to determine the composition of the droplets forming after condensation. Results: The presence of PFO liquid in a closed chamber exacerbates the intensity and likelihood of condensation on all intraocular material types. Condensation of PFO on surfaces in the presence of water was confirmed with FTIR spectroscopy by the isolation of specific absorption bands. Furthermore, material type also affects the characteristics of condensation, with silicone lenses inducing the fastest rate and intensity of condensation.

Correspondence:: Jean-Pierre Hubschman, Stein Eye Institute, David Geffen School of Medicine, University of California, Los Angeles, 100 Stein Plaza Driveway, Los Angeles, CA 90095, USA. E-mail: hubschman@jsei.ucla.edu 
Conclusions: Our study shows that the presence of perfluoro-n-octane is a significant factor in the formation of condensation on the posterior surface of IOLs when performing vitrectomy in a pseudophakic patient with posterior capsulotomy.

\section{2}

\section{Introduction}

The frequency of cataract surgeries has been consistently increasing in the last 20 years. ${ }^{1}$ Projections estimate the total number of persons with cataracts will rise to 30 million by 2020, while the number of pseudophakic/aphakic persons will increase to 9.5 million. ${ }^{1}$ However, cataract surgery is not risk free. It is known that cataract surgery results in a 5.5 times greater risk of developing rhegmatogenous retinal detachment (RRD). ${ }^{2}$ Surgical treatment of RRD frequently involves the use of perfluoro-n-octane liquid (PFO) during pars plana vitrectomy (PPV). In our own surgical experience, use of PFO on pseudophakic patients with a posterior capsulotomy may accelerate IOL surface condensation during fluid-air exchange (FAX), leading to a deteriorated view of the fundus and limiting the surgeon's ability to safely operate.

Condensation on the posterior surface of an IOL during FAX in the presence of a posterior chamber intraocular lens $(\mathrm{PCIOL})$ after posterior capsulotomy or in the presence of an anterior chamber $\mathrm{IOL}(\mathrm{ACIOL})$ has been previously reported. ${ }^{5-12}$ However, there is little data in the literature that addresses whether condensation on IOLs is altered by the presence of PFO. The purpose of this study is to determine whether PFO affects condensation on IOL surfaces.

\section{Materials and methods}

Two separate experiments were carried out. In the first experiment, a high-definition ultra-zoom camera was used to confirm the presence of condensation on three different types of IOLs placed in a closed acrylic and glass chamber. In the second experiment, we employed FTIR spectroscopy to determine the composition of the droplets forming on the surface of the lens.

\subsection{First experiment: high-definition ultra-zoom camera}

Three IOLs were tested, one in polymethylmethacrylate (Multiflex ${ }^{\mathrm{TM}}$ III PMMA Single-Piece IOL, model MTA2UO, Alcon Laboratories, Fort Worth, TX, USA) one in acrylic (ACRYSOF ${ }^{\circledR}$ Single-Piece IOLS, model SA60AT, Alcon Laboratories, Fort Worth, TX, USA), and one in silicone (Elastic Silicone IOL, model AA4204VL, STAAR ${ }^{\circledR}$ SURGICAL, Monrovia, CA, USA). The PFO liquid used was PERFLUORON ${ }^{\circledR}$ Liquid 8065900163 (Alcon Laboratories, Fort Worth, TX, USA).

A chamber was constructed from glass and acrylic panels to serve as an in-vitro eye model. Both chambers were divided into two separate compartments (Fig. 1). 


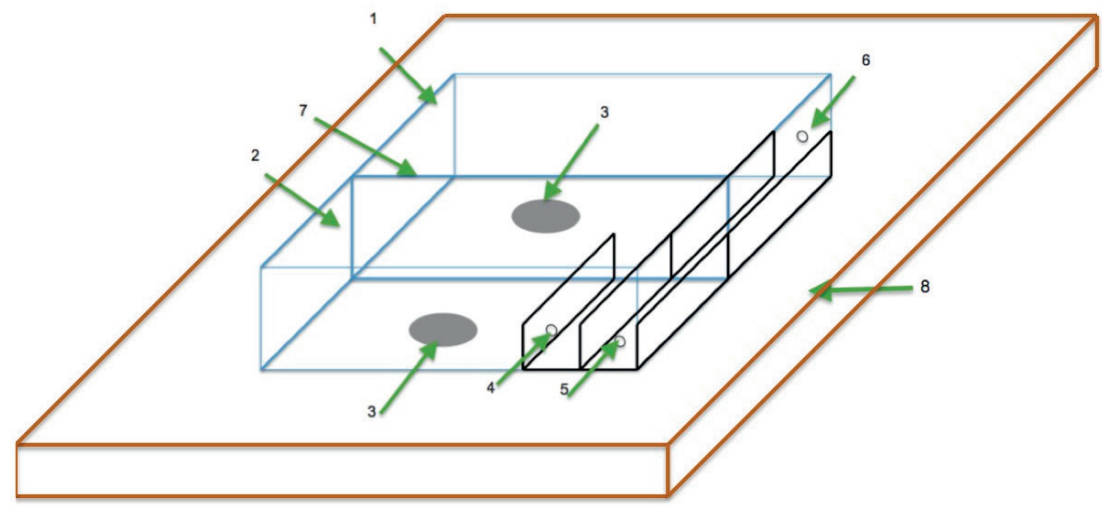

Fig. 1. Scheme of the chamber used in the first experiment. 1. Chamber B; 2. Chamber A; 3 . IOL; 4. PFO dispensary dish injection site/dish; 5 . Water dispensary dish injection site/dish; 6 . Water-only dispensary dish injection site/dish; 7. Chamber divider; 8. Cooling/heating plate.

In the test compartment (chamber A), two small containers were fabricated to accommodate $2.0 \mathrm{ml}$ of water and $2.0 \mathrm{ml}$ of PFO, respectively, with a vacant anterior space for IOL placement. The control compartment (chamber B) had one container, which accommodated $2.0 \mathrm{ml}$ of water, with a vacant anterior space for IOL placement. Two holes were drilled on each side of the chambers to allow for injections of PFO and water into their respective dishes, which were later covered with sealing tape during the trials. The IOLs were placed on a controlled temperature cooling platform, while chamber edges were sealed to assure adequate seal and isolation from the external environment. The chamber was then placed over the IOL, at which time the cooling plate could be activated to a desired temperature. The temperature of the cooling platform (custom built) was monitored by a K-type thermocouple (Model: 39658-K Atkins Technical Inc., Gainesville, FL, USA). A high-definition ultra-zoom camera (LW-1.3-G-1394-M, Imaging Solutions Group, Fairport, NY, USA) was placed above the chamber to record images of the IOL. Recordings of the time of initial condensation and maximum condensation of the IOLs in test and control containers were taken. Prior to the recording of time point data, two observers were asked to run 20 trials to become proficient in recognizing initial and maximum condensation. After each trial, the lenses were plasma cleaned using (Model: PDC-32G, Harrick Plasma, Ithaca, NY, USA). In vitro experiments, IOLs from different materials were tested twice at $10^{\circ} \mathrm{C}, 15^{\circ} \mathrm{C}$, and $20^{\circ} \mathrm{C}$, resulting in 18 trial runs for each chamber and a total of 36 trials. Images were taken, and time points were recorded immediately at the observation of initial and maximum condensation. We measured the relative humidity using a relative humidity sensor (Model: RH32S-C2, Omega, Stamford, CT, USA), finding the test and control containers having humidity levels within $1 \%$ of each other at varying temperatures. Humidity levels for temperatures $10^{\circ} \mathrm{C}, 15^{\circ} \mathrm{C}$, and $20^{\circ} \mathrm{C}$ were $41.0 \%, 50.0 \%$, and $67.0 \%$, respectively. 


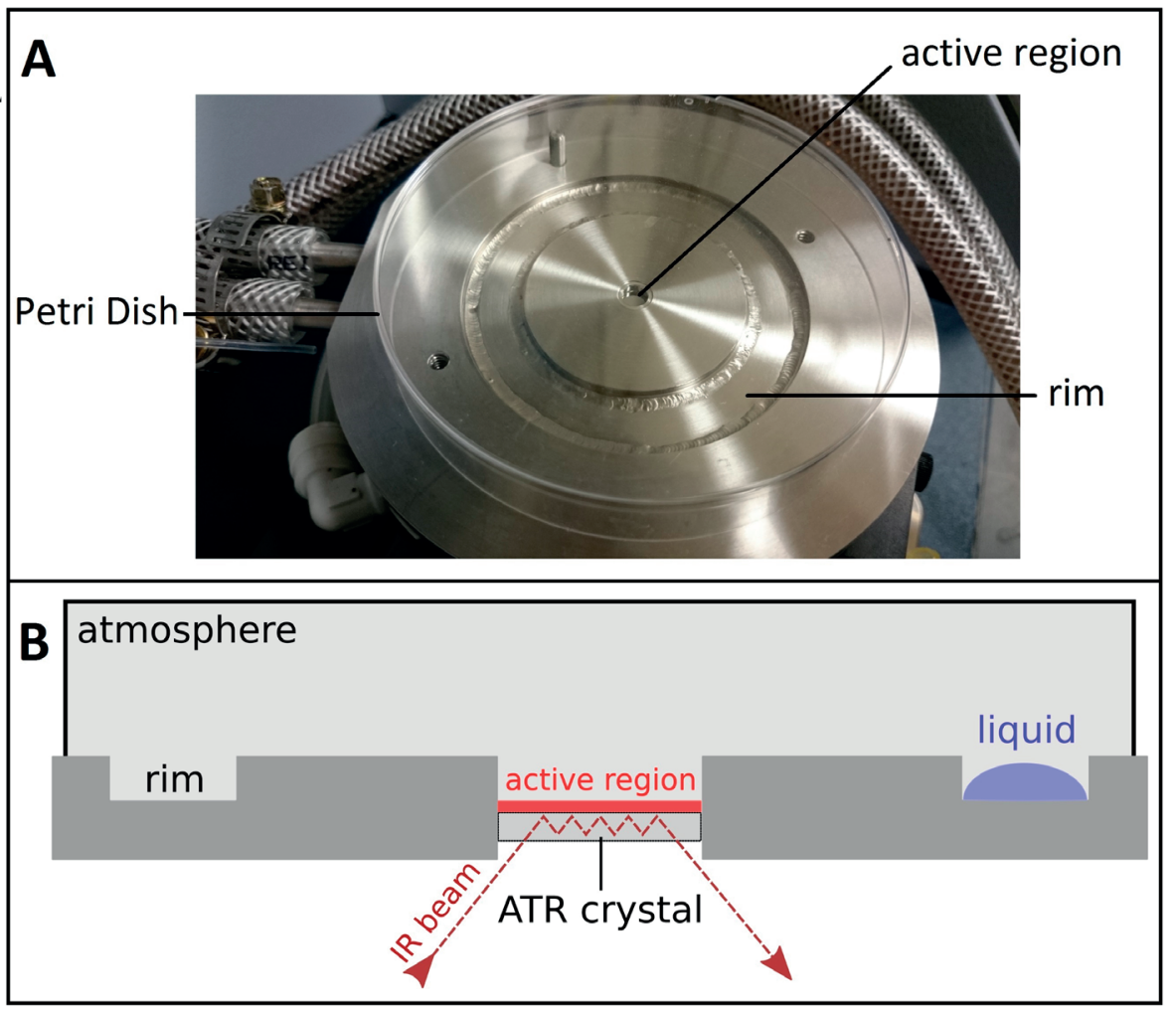

Fig. 2. FTIR spectrometer. $(A)$ Photograph of the rim and active region of the measuring chamber, covered by a Petri dish to create closed and controlled environment. $(B)$ Liquids (PFO and water) were placed either directly on the ATR crystal or in the surrounding rim.

\subsection{Second experiment: FTIR spectroscopy}

FTIR is routinely used to identify compounds based on their molecular vibrations with very high accuracy. ${ }^{13,14}$ The setup used in our investigation is an attenuated total reflection (ATR) system (BioATRCell II, Harrick, NY, USA) and a dry air purged FTIR spectrometer (Tensor 27, Bruker Corp., MA, USA). Fluids can be placed either directly on the ATR crystal or into a rim surrounding the crystal. An atmosphere of approximately $50 \mathrm{~mL}$ volume enclosing crystal and surrounding rim was accomplished using the lid of a Petri dish (Fig. 2).

Reference spectra of water and PFO were recorded placing $20 \mu \mathrm{L}$ of the respective liquid on the ATR crystal. To investigate condensates of vapor formed during the experiment, water, PFO, or both were placed into the surrounding rim. In all FTIR experiments the ATR crystal and surrounding rim was cooled to $15^{\circ} \mathrm{C}$ by a temperature controlled circulating water bath. 


\section{Results}

\subsection{First experiment: high-definition ultra-zoom camera}

In chamber B (water-only chamber), condensation was observed in 0 out of 18 trials for all temperatures and lens materials. In chamber A (PFO and water chamber), condensation was observed in 18 out of 18 trials for all temperatures and material types.

In-vitro findings show that silicone-based lenses consistently had the fastest time of condensation throughout all temperature ranges when compared to PMAA and acrylic lenses. Condensation on silicone lenses at $10^{\circ} \mathrm{C}$ occurred 3.19 minutes prior PMAA and acrylic materials, 3.36 minutes prior at $15^{\circ} \mathrm{C}$, and 8.26 minutes prior at $20^{\circ} \mathrm{C}$. Similarly, maximum condensation occurred faster in silicone IOLs. Disregarding an outlier result of maximum condensation occurring after 45 minutes on an acrylic lens, we found that silicone lenses compared to PMAA and acrylic lenses reached maximum condensation an average of 14.71 minutes earlier at $10^{\circ} \mathrm{C}, 6.44$ minutes earlier at $15^{\circ} \mathrm{C}$, and 3.24 minutes after at $20^{\circ} \mathrm{C}$.

\subsection{Second experiment: FTIR spectroscopy}

Two spectral regions were investigated (Fig. $3 \mathrm{~A}$ and B): Between 3500 and $3000 \mathrm{~cm}^{-1}$, the $\mathrm{O}-\mathrm{H}$ stretching vibration of water causes a broad and intense absorption band (Fig. 3B, blue line). The fingerprint region (1350-1100 $\left.\mathrm{cm}^{-1}\right)$ facilitates unequivocal identification of almost all materials by their specific vibrations; e.g., PFO causes a

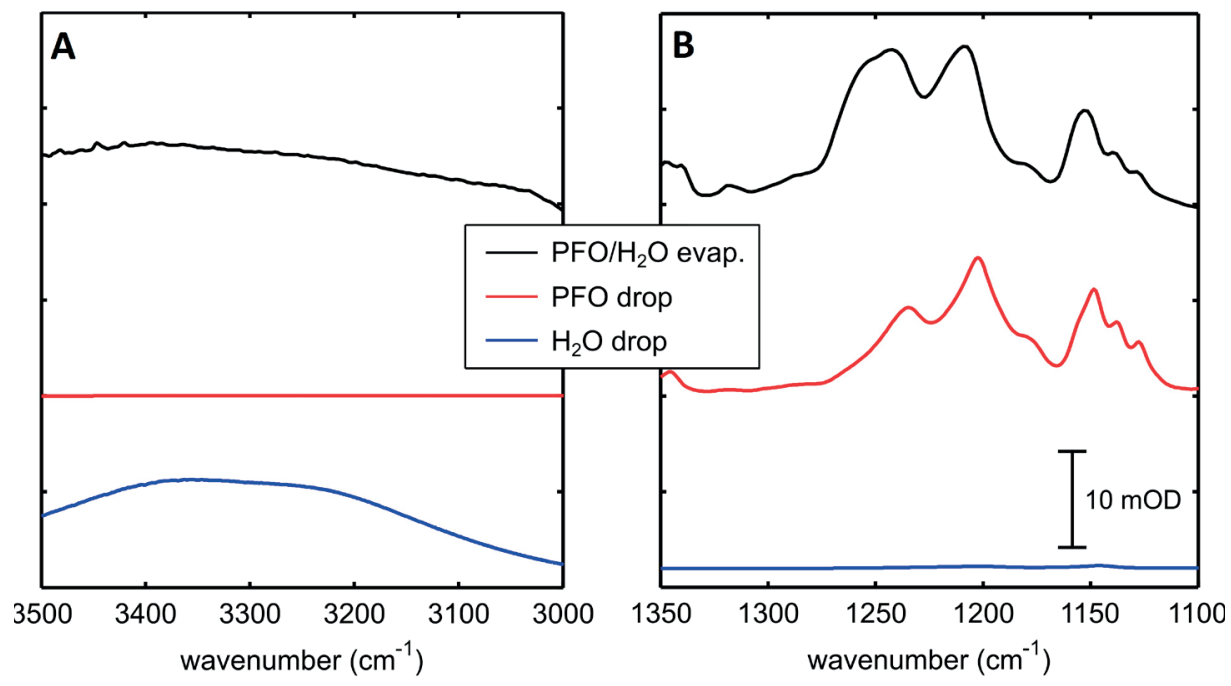

Fig. 3. Spectral regions analyzed. PFO and $\mathrm{H} 2 \mathrm{O}$ absorption bands. (A) Between 3500 and 3000 cm-1 (O-H stretching vibrations). (B) Between 1350 and $1100 \mathrm{~cm}-1$ (fingerprint region). 
characteristic pattern of C-F vibrations when a drop is placed directly on the crystal (red line). When water and PFO are simultaneously placed into the rim surrounding the ATR crystal, after 15 min a combination of both the $\mathrm{H} 2 \mathrm{O}$ and PFO spectra is observed (black line). The above results prove that PFO and water are both present on the ATR crystal after evaporation and condensation of both liquids.

\section{Discussion}

A clear, unobstructed view of the posterior segment is required during retinal surgeries. It has been previously reported that visualization could be compromised by the presence of condensation on the posterior surface of $\mathrm{ACIOL}$ or PCIOL in the presence of a posterior capsulotomy..$^{5-12}$ However, there is little data on whether the presence of PFO may alter the characteristics of condensation. The purpose of this study is to determine if PFO affects condensation on IOL surfaces.

In the present report, it was demonstrated that PFO affects the occurrence, rate, and intensity of condensation on IOL surfaces. Observers were not able to appreciate visible condensation on the IOLs exposed to the water-only chamber, but consistently recorded condensation on the IOLs in the PFO chamber. Furthermore, the study demonstrates that lens material also affects rates and intensities of condensation, with silicone oil lenses being the most conducive in facilitating condensation, which supports previous reports. ${ }^{7}$ The condensation of PFO in presence of water was confirmed by FTIR spectroscopy, which recorded the characteristic spectra of absorption of both PFO and water over the ATR crystal.

Condensation was clearly visible on lenses in the presence of PFO when compared to lenses in the water-only chamber (Fig. 4A and B).

The mechanism for these findings is based on the formation of water vapors and condensation on the IOLs. ${ }^{15-16}$ During film-wise condensation, the interfacial tension
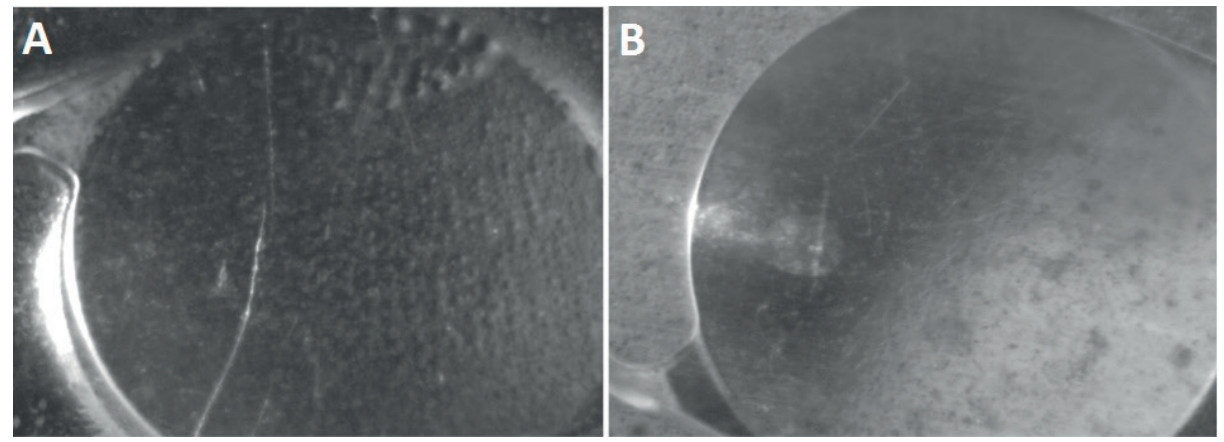

Fig. 4. High-definition photograph of silicone IOL. (A) Silicone lens in the PFO filled chamber at $10^{\circ} \mathrm{C}$ after 20 minutes; condensation is appreciated. $(B)$ Silicone lens in the water-only chamber at $10^{\circ} \mathrm{C}$ after 20 minutes; no condensation can be appreciated. 


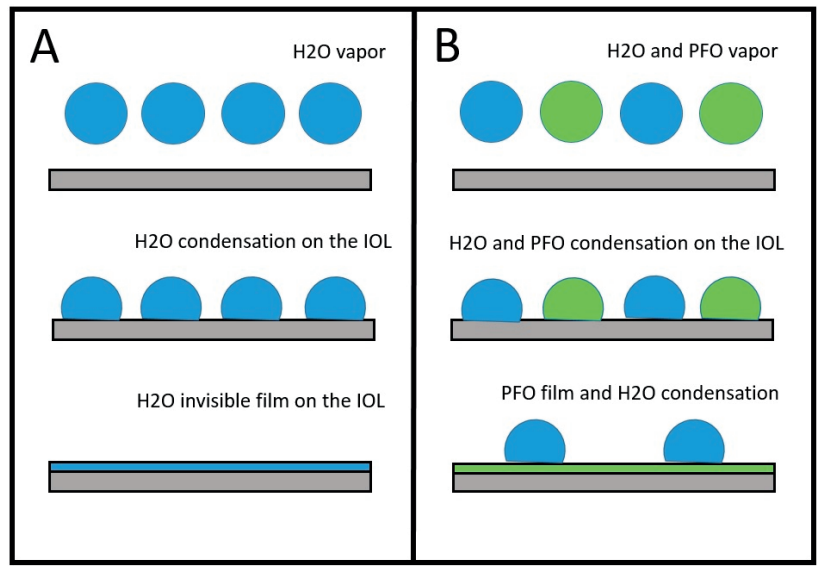

Fig. 5. Interaction between $\mathrm{H}_{2} \mathrm{O}$ and perfluorocarbon at the surface of the IOL. (A) Presence of $\mathrm{H}_{2} \mathrm{O}$ only. $\mathrm{H}_{2} \mathrm{O}$ condensates on the IOL, forming an invisible film without the appearance of fog. (B) Presence of $\mathrm{H} 2 \mathrm{O}$ and PFO. PFO wets on the surface of the lens, forming a thin film due to its very low surface tension. Water cannot wet on the lens and condensates on top the PFO film, causing the fog.

between water and the IOL allows water to fully wet the surface, creating a uniform and invisible film on the surface of the IOL (Fig. 5). ${ }^{11}$

However, water in the presence of PFO cannot form film-wise condensation, resulting in droplet formations on the IOL. The lower surface tension of PFO (14.2$15 \mathrm{dyne} / \mathrm{cm}$ ) allows PFO to preferentially wet the surface of the IOL, which acts as a barrier between IOL surface and water (74dyne/cm). Water vapors form on top of the PFO film, and due to the interactions between water and PFO, droplets are appreciated. It should be noted that the choice of temperatures $\left(10^{\circ} \mathrm{C}, 15^{\circ} \mathrm{C}\right.$, and $20^{\circ} \mathrm{C}$ ) are to show the effect of concurrent condensation of PFO and water on IOL as a function of temperature. The authors tested voluntary lower temperature than found in classic clinical settings to "expedite" the formation of fog; the lower IOL temperatures produce larger drops at faster rates. However, the physics of the fogging process remains the same. These findings are consistent with our intraoperative experience, in which IOL condensation occurs more easily when PFO is being used.

Different lens materials have different interfacial relationships with PFO and water, resulting in varying intensities of condensation. The varying rates and intensities of condensation found on PMMA, acrylic, and silicone IOLs are due to the differences in the materials surface free energy. Surface free energy can be measured with contact angle as a means to quantify hydrophobicity. Contact angle is defined as the angle between the material surface and a tangent on the surface of a droplet. Silicone $\left(114.1^{\circ}\right)$ has the largest contact angle, followed by hydrophobic acrylic $\left(88.7^{\circ}\right)$ and PMMA $\left(77.1^{\circ}\right) .{ }^{17}$ Our findings indicate that materials with greater 
surface free energy correlate with greater intensities of condensation. Silicone has the greatest rate and intensity of condensation, followed by acrylic and PMMA lenses. Based on our findings, PFO has the potential to exacerbate condensation on acrylic, silicone, and PMAA IOLs by acting as a buffer between water droplets and the surface of the IOL, not allowing film-wise condensation. We conclude that PFO increases the risk of condensation on the posterior surface of IOLs, regardless of material type. These findings are relevant, as surgeons utilizing PFO in pseudophakic patients with posterior capsulotomies increase the risk of operating with a deteriorated view of the fundus, which can lead to intraoperative complications.

\section{Summary}

Condensation on the posterior surface of an IOL in the presence of a posterior capsulotomy results in a deteriorated view of the retina. A diminished view of the retina can result in surgical complications, as unobstructed visualization of the posterior segment of the eye is required in various retinal surgeries. The results of this study indicate that there is a significant correlation between the presence of PFO and an increased occurrence and intensity of condensation. Furthermore, our study demonstrates that silicone IOLs are the most conducive lenses for condensation, which supports the findings of previous studies. Future studies could employ mathematical modeling in identifying, quantifying, and characterizing the surgical conditions and material properties that will help reduce surgical complications associated with PFO and IOLs.

\section{Acknowledgements}

None of the authors have proprietary interest. 


\section{References}

1. Congdon N, Vingerling JR, Klein BE, et al. Eye Diseases Prevalence Research Group. Prevalence of cataract and pseudophakia/aphakia among adults in the United States. Arch Ophthalmol. 2004;122(4):487-494.

2. Rowe JA, Erie JC, Baratz KH, et al. Retinal detachment in Olmsted County, Minnesota, 1976 through 1995. Ophthalmology. 1999;106:154-159.

3. Brazitikos PD, Androudi S, D’Amico DJ, MD, et al. Perfluorocarbon liquid utilization in primary vitrectomy repair of retinal detachment with multiple breaks. Retina. 2003;23(5):615-21.

4. Chang S, Ozmert E, Zimmerman NJ, et al. Intraoperative perfluorocarbon liquids in the management of proliferative vitreoretinopathy. Am J Ophthalmol. 1998;106: 668-674.

5. Brazitikos, PR. Perfluorocarb uid utilization in primary vitrectomy repair of

6. retinal detachment with mul 2 breaks. Retina. 2003; 23:615-621.

7. Eaton AM, Jaffe GJ, McCuen BW 2nd, et al. Condensation on the posterior surface of silicone intraocular lenses during fluid-air exchange. Ophthalmology. 1995;102(5):733-736.

8. Hainsworth DP, Chen SN, Cox TA, et al. Condensation on polymethylmethacrylate, acrylic polymer, and silicone intraocular lenses after fluid-air exchange in rabbits. Ophthalmology. 1996;103(9):140148.

9. Jaffe GJ. Management of condensation on a foldable acrylic intraocular lens after vitrectomy and fluid-air exchange. Am J Ophthalmol. 1997;124:692-693.

10. Kusaka S, Kodama T, Ohashi Y. Condensation of silicone oil on the posterior surface of a silicone intraocular lens during vitrectomy. Am J Ophthalmol. 1996;121:574-575.

11. Eaton AM, Jaffe GJ, McCuen BW II, et al. Condensation on the posterior surface of silicone intraocular lenses during fluid-air exchange. Ophthalmology. 1995;102:733-736.

12. Porter RG, Peters JD, Bourke RD. De-misting condensation on intraocular lenses. Ophthalmology. 2000;107:778-782.

13. Browning DJ, Fraser CM. Clinical management of silicone intraocular lens condensation. Am J Ophthalmol. 2005;139(4):740-742.

14. Stuart BH. In Infrared spectroscopy: fundamentals and applications. John Wiley \& Sons, Inc.; 2005:71-93.

15. Stuart BH. In Infrared spectroscopy: fundamentals and applications. John Wiley \& Sons, Inc.; 2005;95-111.

16. Moran MJ, Shapiro HN, Boettner DB, Bailey MB. Fundamentals of engineering thermodynamics. Dec 2010 Wiley; 7th edition

17. Bergman TL, Lavine AS, Incropera FP, et al. Fundamentals of heat and mass transfer. April 2011. Wiley; 7th edition.

18. Baillif S, Baziard-Mouysset G, Roques C, et al. Calculation of intraocular lens surface free energy and its components from contact angle measurements. Ophthalmic Res. 2013;50(3):165-173. 
